\title{
Anisotropic colloidal particles interacting with polymers in a good solvent
}

\author{
E. Eisenriegler \\ Institut für Festkörperforschung, \\ Forschungszentrum Jülich, \\ D-52425 Jülich, Germany
}

(Dated: February 7, 2006)

\begin{abstract}
We study the consequences of chain self-avoidance for the interaction between nonadsorbing polymers and colloidal particles of anisotropic shape, such as ellipsoids, lenses, and dumbbells. In the framework of a field theoretic operator-expansion for small mesoscopic particles, we obtain exact results for self-avoiding polymers in $d=2$ spatial dimensions, and we compare ideal and self-avoiding polymers in $2<d<4$. Changing the orientation of a particle, with a given size and shape, near a boundary wall requires less free energy if the chains are self-avoiding. The distancedependence of the anisotropic interaction, mediated by the polymers between particle and wall, is changed both qualitatively and quantitatively by self-avoidance.
\end{abstract}




\section{INTRODUCTION}

The depletion interaction between colloidal particles in a solution of polymers is one of the basic interactions in soft matter composite materials ${ }^{1}$. Free nonadsorbing polymer chains avoid the space between two particles, leading to an unbalanced pressure, which pushes the particles towards each other. The depletion forces between a pair of immersed particles and between a single particle and a wall have been measured in recent experiments ${ }^{2}$.

While the induced interaction between mesoscopic particles in a dilute or semidilute solution of long flexible polymers is independent of most microscopic details, it does depend on the quality of the solvent (good or theta solvent) and the size and shape of the particles.

Here we consider particles of anisotropic shape, for which the interaction depends on both the distances between particles and their mutual orientations. Thus, the entropic polymerinduced interaction provides both a force and a torque. For simplicity we concentrate on particle shapes with an axis of rotational symmetry and a center of reflection, such as ellipsoids, lenses, or dumbbells ${ }^{3}$, in a dilute polymer solution.

For mesoscopic particles much smaller than the polymer lengths, such as ${ }^{4}$ the root mean square end-to-end distance $\mathcal{R}_{e}$, the perturbation of the polymer system due to a particle can be expanded in powers of the particle size. The two leading orientation-dependent terms vary as (size $)^{x}$ and (size) $)^{x^{\prime}}$ with exponents ${ }^{5}$

$$
x=d \quad, \quad x^{\prime}=d-(1 / \nu)+2,
$$

where $d$ is the spatial dimension and $\nu$ the Flory exponent relating the end-to-end distance $\mathcal{R}_{e} \propto M^{\nu}$ of a long polymer chain to the number of monomers $M$. Besides the size exponents the perturbation of the polymer system is characterized by universal amplitudes that depend on the shape of the particle.

The orientation-dependent interactions induced by ideal, random-walk-like polymers ${ }^{6}$ have been discussed in Refs. 5 and 7. In this case $\nu=1 / 2$, the two exponents in (1.1) are equal, and the two contributions of order $(\text { size })^{x}$ and $(\text { size })^{x^{\prime}}$ are of equal importance. Together they lead to an induced interaction between a particle and a planar wall favoring parallel orientation for small particle-wall distances $z \ll \mathcal{R}_{e}$ but perpendicular orientation for large distances $z \gg \mathcal{R}_{e}$. For self-avoiding polymers in a good solvent, one expects qualitative modifications of the ideal chain results, since $\nu>1 / 2$ and $x<x^{\prime}$. Thus, the degeneracy 
of the exponents is removed, and the orientation-dependent properties are dominated by the contribution $\propto(\text { size })^{x}$, while the contribution $\propto(\text { size })^{x^{\prime}}$ can in general be neglected.

The above expansion can be understood as an 'operator-expansion' ${ }^{8,9}$ of the Boltzmann weight of the embedded particle in a field theory that corresponds to the polymer system

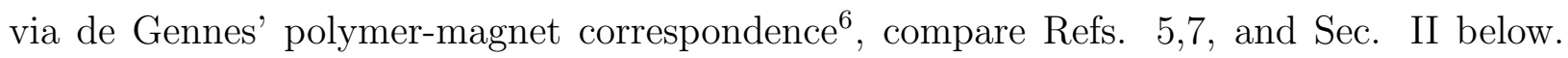
In the field theory the perturbation due to the small anisotropic mesoscopic particle can be represented by a series of isotropic and anisotropic point operators. This 'small-particle expansion' is analogous to the multipole expansion for a localized charge distribution or to the well known short-distance expansion of an operator product in field theory. The leading anisotropic operator is the diagonal stress-tensor component $T_{\|\|}$along the symmetry axis of the particle which has the scaling dimension $x=d$.

The small-particle expansion and polymer-magnet correspondence is introduced in Sec. II. In Sec. III we consider the operator expansion in the $N$-vector field theory and calculate the shape-dependent amplitudes of the leading isotropic and anisotropic operators for dumbbells, lenses, and ellipses in $d=2$, and for a dumbbell of two touching spheres in $d=4-\varepsilon$. We also consider weakly anisotropic particles of a more general shape. In Sec. IV we use properties of the stress tensor to evaluate the polymer-induced orientation-dependent interaction in a good solvent between an anisotropic particle and a planar wall. The results are summarized in Sec. V. Somewhat more technical material is presented in the three Appendices.

\section{PARTICLE-POLYMER INTERACTION AND SMALL-PARTICLE EXPANSION IN THE $N$-VECTOR MODEL}

A nonadsorbing polymer interacting with a colloidal particle corresponds ${ }^{6,7,10}$ to an $N$ vector field theory outside a particle with an 'ordinary' surface ${ }^{11,12}$, which favors disorder and preserves the $O(N)$ symmetry of the $N$-vector order parameter. In the field theory the Boltzmann factor $\exp (-\delta \mathcal{H})$ of a particle at $\mathbf{r}_{\mathrm{P}}$ can be expanded as ${ }^{8,9}$

$$
e^{-\delta \mathcal{H}} \propto 1-s_{I}-s_{A}
$$

in terms of isotropic

$$
s_{I}=\mathcal{I} \psi\left(\mathbf{r}_{\mathrm{P}}\right)+\mathcal{I}^{\prime} \Delta \psi\left(\mathbf{r}_{\mathrm{P}}\right)+\ldots
$$


and anisotropic operators

$$
s_{A}=\sum_{k l}\left[\rho_{k} \rho_{l}-\left(\delta_{k l} / d\right)\right]\left[\mathcal{N} T_{k l}\left(\mathbf{r}_{\mathrm{P}}\right)+\mathcal{N}^{\prime} \partial_{k} \partial_{l} \psi\left(\mathbf{r}_{\mathrm{P}}\right)\right]+\ldots
$$

from the operator algebra that are compatible with the particle symmetries. Here $T_{k l}$ is the stress tensor of the field theory, $\vec{\rho}$ is the unit vector along the symmetry axis of the particle, and $\sum_{k l} \rho_{k} \rho_{l} T_{k l}$ and $\sum_{k l} \rho_{k} \rho_{l} \partial_{k} \partial_{l}$ will be denoted by $T_{\|\|}$and $\partial_{\|}^{2}$, respectively. There is no first derivative, due to reflection symmetry. The leading isotropic operator $\psi$ is proportional to the energy density $\epsilon$, and a convenient normalization is ${ }^{13}$

$$
\psi=-\mathcal{A}_{\epsilon} \epsilon / B_{\epsilon}^{1 / 2}
$$

where $B_{\epsilon}$ and $\mathcal{A}_{\epsilon}$ are the amplitudes in the bulk two-point function $\langle\epsilon(\mathbf{r}) \epsilon(\mathbf{0})\rangle_{\text {bulk }}=B_{\epsilon} r^{-2 x_{\epsilon}}$ and the half space ('hs') profile $\left\langle\epsilon\left(\mathbf{r}_{p}, z\right)\right\rangle_{\mathrm{hs}} / B_{\epsilon}^{1 / 2}=\mathcal{A}_{\epsilon} z^{-x_{\epsilon}}$ at the critical point. In the half space, $\mathbf{r}_{p}$ and $z$ denote the components of $\mathbf{r}$ parallel and perpendicular to the planar boundary. $B_{\epsilon}$ and $\mathcal{A}_{\epsilon}$ are positive for $N>0$. The scaling dimension of $\epsilon$ and $\psi$ is $x_{\epsilon}=$ $d-(1 / \nu)$, while the stress tensor has scaling dimension $d$. Both $\psi$ and $T_{k l}$ are $O(N)$-invariant operators. We note that the bulk two-point function $\left\langle\epsilon T_{k l}\right\rangle$ vanishes at the critical point. The size powers in the shape-dependent prefactors $\mathcal{I}, \mathcal{I}^{\prime}, \mathcal{N}, \mathcal{N}^{\prime}$ in (2.2) and (2.3) are given by the scaling dimensions $x_{\epsilon}, x_{\epsilon}+2, d, x_{\epsilon}+2$, respectively, of the corresponding operators ${ }^{14}$.

The form of the prefactors is different for ideal and self-avoiding chains. Since $\mathcal{I}$ is proportional to $(\text { size })^{x_{\epsilon}}$, the expansion only makes sense for $x_{\epsilon}>0$. For ideal chains with $x_{\epsilon}=d-2$ this means $d>2$. For self-avoiding chains, on the other hand, there is a meaningful expansion in $d=2$, since $\nu=3 / 4$ and $x_{\epsilon}=2 / 3$.

Single-chain properties follow from correlation functions in the high temperature ('paramagnetic') phase of the $N$-vector field theory by means of an inverse Laplace transform

$$
\mathcal{L} \ldots=\int(\mathrm{d} t /(2 \pi i)) e^{L_{\mathrm{pol}} t} \ldots
$$

where $L_{\text {pol }}$ is proportional to the length (number $M$ of monomers) of the polymer chain, and the Laplace conjugate $t$ relates the density of the thermal perturbation,

$$
\mathcal{T}(\mathbf{r})=-t \epsilon(\mathbf{r})
$$

in the $N$-vector Hamiltonian to the energy-density operator $\epsilon$ and is proportional to the deviation from the critical temperature ${ }^{6}$. 
Consider, for example, the free energy cost $F$ of immersing particles of arbitrary size in a dilute solution of self-avoiding polymers in an unbounded space or in the half space bounded by a wall 7 . This is given by

$$
F=-p_{0} \frac{\int \mathrm{d} \mathbf{r}_{1} \mathrm{~d} \mathbf{r}_{2} \mathcal{L}\left[\left\langle\varphi_{12}\right\rangle_{\mathcal{H}+\delta \mathcal{H}}-\left\langle\varphi_{12}\right\rangle_{\mathcal{H}}\right]}{\int \mathrm{d} \mathbf{r}_{1} \mathcal{L}\left\langle\varphi_{12}\right\rangle_{\text {bulk }}} .
$$

Here $p_{0}=n k_{B} T$ is the ideal gas pressure in the dilute solution with chain density $n$, and $\varphi_{12}$ denotes the scalar product $\vec{\Phi}\left(\mathbf{r}_{1}\right) \vec{\Phi}\left(\mathbf{r}_{2}\right)$ of two $N$-vector order parameter fields $\vec{\Phi}$. The subscripts $\mathcal{H}+\delta \mathcal{H}$ and $\mathcal{H}$ denote $N$-vector Hamiltonians in the presence and absence of the particles, respectively, and the subscript 'bulk' denotes averaging in the unbounded space, without the particles and the wall. Finally it is understood that the limit $N \rightarrow 0$ is taken ${ }^{6}$ on the right hand side of (2.7). A universal expression for $F$, independent of the chain microstructure, is obtained in the scaling limit by expressing $L_{\text {pol }}$ in terms of ${ }^{4}$ the mean square end-to-end distance $\mathcal{R}_{e}^{2}=d \mathcal{R}_{x}^{2}$, given by $\int \mathrm{d} \mathbf{r}_{12}\left(\mathbf{r}_{12}\right)^{2} \mathcal{L}\left\langle\varphi_{12}\right\rangle_{\text {bulk }} / \int \mathrm{d} \mathbf{r}_{12} \mathcal{L}\left\langle\varphi_{12}\right\rangle_{\text {bulk }} \propto$ $L_{\mathrm{pol}}^{2 \nu}$, where $\mathbf{r}_{12}=\mathbf{r}_{1}-\mathbf{r}_{2}$.

In Sec. III we consider the expansion (2.1)-(2.3) in detail and calculate the universal amplitudes $\mathcal{I}, \mathcal{I}^{\prime}, \mathcal{N}, \mathcal{N}^{\prime}$ for various particle shapes, spatial dimensions $d$, and numbers $N$ of components. In the limit $N \rightarrow 0$, corresponding to self-avoiding polymers, the amplitudes remain finite ${ }^{15}$, and

$$
2^{x_{\epsilon}} \psi\left(\mathbf{r}_{P}\right) \rightarrow A \Psi\left(\mathbf{r}_{P}\right)
$$

in terms of the monomer density operator $\Psi(\mathbf{r})$ satisfying $^{10,16,17}$

$$
\frac{\int \mathrm{d} \mathbf{r}_{1} \mathrm{~d} \mathbf{r}_{2} \mathcal{L}\left\langle\Psi\left(\mathbf{r}_{p}, z\right) \varphi_{12}\right\rangle_{\mathrm{hs}}}{\int \mathrm{d} \mathbf{r}_{1} \mathcal{L}\left\langle\varphi_{12}\right\rangle_{\text {bulk }}}=\mathcal{R}_{x}^{1 / \nu} \mathcal{M}_{\mathrm{hs}}\left(z / \mathcal{R}_{x}\right),
$$

where $\mathcal{M}_{\mathrm{hs}}$ is the bulk-normalized monomer density profile of a dilute polymer solution in the half space and $z$ the distance from its boundary wall. Compare also the "normalization property' given in Eq. (C2) below. In Eq. (2.8) $A$ is the universal amplitude in the free energy cost

$$
F_{\text {bulk }}^{\text {(sphere) }}=p_{0} A R^{x_{\epsilon}} \mathcal{R}_{x}^{1 / \nu}
$$

of immersing a small spherical particle of radius $R$ in the bulk solution. In this case $\mathcal{I} / 2^{x_{\epsilon}}=$ $R^{x_{\epsilon}}$, see Ref. 8. Results for $A$ in various spatial dimensions $d$ are given in Table I of Ref. 17 where $A$ is denoted by $A_{D, D}$. A relation between the universal amplitudes $A$ and $\mathcal{A}_{\epsilon}$ is given in Eq. (C5) below. 
Ideal chains are related ${ }^{7}$ to a one-component Gaussian model. Within the $N$-vector model this corresponds to the special case of vanishing anharmonic interactions in which it reduces to $N$ independent one-component Gaussian models. Then the right hand side of Eq. (2.7) becomes independent of $N$ and describes the free energy $F=F_{\text {ideal }}$ of ideal chains. Likewise $x_{\epsilon}, B_{\epsilon} / N$ and $\mathcal{A}_{\epsilon} / \sqrt{N}$ become independent of $N$. Anharmonicity-contributions to $\left\langle\varphi_{12}\right\rangle$ carry combinatorial factors in perturbation theory which vanish for $N \rightarrow-2$. Thus, even in the general case of nonvanishing anharmonicities the right hand side of (2.7) reduces to the ideal chain result if $N \rightarrow-2$.

In evaluating the polymer-induced particle-wall interaction (2.7) by means of the expansion (2.1) for a small particle, the contributions from the $\mathcal{I}, \mathcal{I}^{\prime}$, and $\mathcal{N}^{\prime}$ terms in (2.2), (2.3) follow from Eqs. (2.8) and (2.9). The contribution from the stress tensor term proportional to $\mathcal{N}$ will be given in Sec. IV.

\section{SMALL PARTICLE AMPLITUDES}

The amplitudes $\mathcal{I}, \mathcal{I}^{\prime}, \mathcal{N}, \mathcal{N}^{\prime}$ in Eqs. (2.2) and (2.3) depend on the size and shape of the particle. Since they are independent ${ }^{8,9}$ of other particles or a distant wall and of $t$, they follow most easily from the density profiles $\langle\psi\rangle_{\text {part }},\left\langle T_{k l}\right\rangle_{\text {part }}$ induced by a single particle in the $N$-vector model, right at the critical point. We will check that the same amplitudes reproduce two-point functions such as $\langle\psi \psi\rangle_{\text {part }}$ at large distances from the particle.

Our main interest is in particles with the shapes of ellipsoids, lenses, and dumbbells. The ellipsoids have diameters $D_{\|}$and $D_{\perp}$ parallel and perpendicular to the rotation axis $\vec{\rho}$ and are prolate, $D_{\|}>D_{\perp}$, or oblate, $D_{\|}<D_{\perp}$. We consider dumbbells of two intersecting spheres with sphere-diameter $L$, angle of intersection $\alpha$, and diameter $\mathcal{D}=L \sin (\alpha / 2)$ of the circle of intersection, as in Fig. 1 of Ref. 5 or the inset of Fig. 2 below. The symmetry axis containing the unit vector $\vec{\rho}$ is the straight line passing through the centers of the two spheres. For $\alpha=0$ the dumbbell consists of two touching spheres with $\mathcal{D}=0$, and for $\alpha=\pi$ it reduces to a single sphere with diameter $L=\mathcal{D}$. By further increasing $\alpha$ one obtains lenses with spherical surfaces, which for $\alpha=2 \pi$ reduce to a spherical disk of diameter $\mathcal{D}$, see the inset of Fig. 1 below. 


\section{A. Ellipses, lenses and dumbbells in two dimensions}

In two spatial dimensions, the critical profiles and correlation functions induced by a single particle of arbitrary shape follow from those induced by the straight boundary of the half plane by means of an appropriate conformal transformation ${ }^{18}$. Here we consider the leading and next to leading anisotropic orders in the small particle expansion, corresponding to operators of scaling dimensions $d=2$ and $x_{\epsilon}+2$ for ellipses, lenses, and dumbbells. Besides $\partial_{\|}^{2} \psi$, there exists in two dimensions another ${ }^{19} \mathrm{O}(N)$-symmetric and spatially anisotropic operator,

$$
\tilde{\psi}=-\left(\rho_{+}^{2} \Lambda+\rho_{-}^{2} \bar{\Lambda}\right) \psi
$$

with symmetry axis $\vec{\rho}=\left(\rho_{x}, \rho_{y}\right)$ in the $x, y$ plane and scaling dimension $x_{\epsilon}+2$. This contributes a term

$$
\mathcal{N}^{\prime \prime} \tilde{\psi}\left(\mathbf{r}_{\mathrm{P}}\right)
$$

to the right hand side of (2.3). Here

$$
\rho_{ \pm}=\rho_{x} \pm i \rho_{y}
$$

and

$$
\Lambda \mathcal{O}=\left[L_{-2}-\frac{3}{2\left(x_{\mathcal{O}}+1\right)} L_{-1}^{2}\right] \mathcal{O}
$$

for a primary operator $\mathcal{O}$ such as the energy density $\epsilon$ or $\psi$. The operators $L_{-1} \mathcal{O} \equiv$ $\partial_{w} \mathcal{O}, \bar{L}_{-1} \mathcal{O} \equiv \partial_{\bar{w}} \mathcal{O}$, with $w=r_{x}+i r_{y}, \bar{w}=r_{x}-i r_{y}$, and $L_{-2} \mathcal{O}, \bar{L}_{-2} \mathcal{O}$ appear in the operator-product expansion ${ }^{19}$ of $\mathcal{O}$ and $T(w) \equiv-\pi\left(T_{x x}-i T_{x y}\right)$ or $\bar{T}(\bar{w}) \equiv-\pi\left(T_{x x}+i T_{x y}\right)$.

The anisotropic operators $T_{\|\|}, \partial_{\|}^{2} \psi$, and $\tilde{\psi}$ are mutually 'orthogonal', i.e. the bulk twopoint functions of different operators vanish at the critical point. In particular, $\tilde{\psi}$ is orthogonal to $\psi$ and its derivatives. In the case $N=1$ of the Ising model, $\tilde{\psi}$ appears neither in the operator algebra ${ }^{19}$ nor in small particle expansions ${ }^{9}$, since it vanishes in any correlation function.

The results in Appendix A yield for an ellipse

$$
\mathcal{I}=\mathcal{C}^{x_{\epsilon}} \quad, \quad \mathcal{I}^{\prime}=\mathcal{C}^{x_{\epsilon}+2} /\left(16 x_{\epsilon}\right)
$$




$$
\mathcal{N}=-\mathcal{C}^{2}(\pi / 2) g \quad, \quad \mathcal{N}^{\prime \prime}=\mathcal{C}^{x_{\epsilon}+2} g / 4
$$

where

$$
\mathcal{C} \equiv\left(D_{\perp}+D_{\|}\right) / 2 \quad, \quad g \equiv\left(D_{\perp}-D_{\|}\right) /\left(D_{\perp}+D_{\|}\right)
$$

and for the lens and dumbbell

$$
\begin{gathered}
\mathcal{I}=(\mathcal{D} \pi / \alpha)^{x_{\epsilon}} \quad, \quad \mathcal{I}^{\prime}=(\mathcal{D} \pi / \alpha)^{x_{\epsilon}+2} /\left(16 x_{\epsilon}\right), \\
\mathcal{N}=-\mathcal{D}^{2}(\pi / 6)\left[1-(\pi / \alpha)^{2}\right] \\
\mathcal{N}^{\prime \prime}=\mathcal{D}^{x_{\epsilon}+2}(\pi / \alpha)^{x_{\epsilon}}\left[1-(\pi / \alpha)^{2}\right] / 12 .
\end{gathered}
$$

For both families of particles,

$$
\mathcal{N}^{\prime}=-\frac{3}{2\left(x_{\epsilon}+1\right)} \mathcal{N}^{\prime \prime}
$$

so that $\mathcal{N}^{\prime} / \mathcal{N}^{\prime \prime}$ is independent of the particle shape. The prefactor $\mathcal{N}$ of the stress tensor in (3.6) and (3.9) is independent of $N$ and equals the limit of $\mathcal{N}=\mathcal{N}_{\text {ideal }}$ for ideal chains ${ }^{5,9}$ as $d \searrow 2$. For dumbbells with $\alpha=0$ and $\alpha=\pi / 2$ see Eq. (3.15) below and Eq. (B11) in Ref. 5. The exponent $x_{\epsilon}$ and the amplitude combination $\mathcal{A}_{\epsilon}^{2} / N$ are $\mathrm{an}^{20}$ analytic functions of $N$ with the values $0,2 / 3,1,2$ and $1 / 2,2^{2 / 3} / 3^{3 / 2}, 1 / 4,3 / 2^{5}$ for $N=-2,0,1,2$, respectively. In Appendix A we compare the small-particle expansion considered here with the usual short-distance expansion of an operator-product ${ }^{19}$.

The ellipse and lens expressions coincide, as they must, for the circle of radius $R$ with $D_{\perp}=D_{\|}=2 R, \alpha=\pi, \mathcal{D}=2 R$, and for the needle of length $l$ perpendicular to the axis $\vec{\rho}$ with $D_{\perp}=l, D_{\|}=0, \alpha=2 \pi, \mathcal{D}=l$. The needle in $d=2$ corresponds to the spherical disk mentioned above Sec. IIIA.

The corresponding anisotropy amplitudes of the ellipse and lens/dumbbell families have the same sign if $g$ and $1-(\pi / \alpha)^{2}$ are positive, i.e. for particles more extended perpendicular to $\vec{\rho}$ than parallel to it, and the opposite sign if $g$ and $1-(\pi / \alpha)^{2}$ are negative.

It is instructive to compare the leading anisotropy amplitude $\mathcal{N}$ of a lens or dumbbell with that of the smallest circumscribing ellipse (CE). For the lens with $\alpha=2 \pi-$ $\alpha^{\prime}, 0 \leq \alpha^{\prime} \leq \pi$, the $\mathrm{CE}$ has diameters $D_{\perp}=\mathcal{D}, D_{\|}=\mathcal{D} \operatorname{tg}\left(\alpha^{\prime} / 4\right)$, and its amplitude $\mathcal{N}_{\mathrm{CE}}=-(\pi / 8) \mathcal{D}^{2} \cos \left(\alpha^{\prime} / 2\right) / \cos ^{2}\left(\alpha^{\prime} / 4\right)$ has a larger modulus than the amplitude 
$\mathcal{N}_{\text {lens }}=-(\pi / 8) \mathcal{D}^{2}\left(\pi-\alpha^{\prime} / 3\right)\left(\pi-\alpha^{\prime}\right) /\left(\pi-\alpha^{\prime} / 2\right)^{2}$ of the lens in Eq. (3.9), see Fig. 1. For the dumbbell with $0 \leq \alpha \leq \pi$, the CE has diameters $D_{\perp}=\sqrt{2} L \cos (\alpha / 4), D_{\|}=2 L \cos ^{2}(\alpha / 4)$, and its amplitude $\mathcal{N}_{\mathrm{CE}}=(\pi / 4) L^{2} \cos ^{2}(\alpha / 4) \cos (\alpha / 2)$ is smaller than the amplitude $\mathcal{N}_{\text {dumb }}=$ $(\pi / 6) L^{2} \sin ^{2}(\alpha / 2)\left[(\pi / \alpha)^{2}-1\right]$ of the dumbbell in Eq. (3.9), see Fig. 2. To the self-avoiding polymers the lens and dumbbell thus appear less and more anisotropic, respectively, than their circumscribing ellipses. This is expected intuitively, and in line with similar findings ${ }^{5,7}$ for ideal polymers in $d=3$.

\section{B. Dumbbell of two touching spheres in $d=4-\varepsilon$}

Consider the dumbbell of two touching spheres, each of diameter $L$. The particle amplitudes follow from profiles in the parallel plate (film) geometry by an inversion about a midpoint of the film ${ }^{9}$. Using the results of Refs. 22, 23, and 24 for the energy density and stress tensor profiles in a film and the stress-tensor two-point function in the bulk, in $d=4-\varepsilon$ spatial dimensions, we find ${ }^{25}$

$$
\begin{aligned}
& \mathcal{I}=(L / 2)^{x_{\epsilon}} 4\left(2^{d-3}-1\right) \zeta(d-2)\left\{1+\varepsilon \kappa[\ln \pi-(1 / 4)]+O\left(\varepsilon^{2}\right)\right\} \\
& \mathcal{I}^{\prime}=\frac{2\left(x_{\epsilon}+1\right) / d}{2\left(x_{\epsilon}+1\right)-d} \mathcal{N}^{\prime} \\
& \mathcal{N}^{\prime}=(L / 2)^{x_{\epsilon}} L^{2} \frac{1}{8 x_{\epsilon}\left(x_{\epsilon}+1\right)} \frac{1}{2}(d-2)(d-1)\left(2^{d}-1\right) \zeta(d) \\
& \quad \times\left\{1+\varepsilon \kappa[\ln \pi+(1 / 3)]+O\left(\varepsilon^{2}\right)\right\}
\end{aligned}
$$

and

$$
\mathcal{N}=(L / 2)^{d} 4(\pi / 4)^{d / 2}(d-1)[\zeta(d) / \Gamma(d / 2)]\left\{1-5 \varepsilon \kappa / 4+O\left(\varepsilon^{2}\right)\right\}
$$

where

$$
\kappa=(N+2) /(N+8)
$$

and $\zeta$ is the Riemann zeta function. For $N=0$ the right hand sides of Eqs. (3.12)-(3.15) determine the behavior of self-avoiding chains, while for $N=-2$, where $x_{\epsilon}=d-2, \kappa=0$, and the curly brackets equal 1 , they reduce to expressions ${ }^{9}$ of the Gaussian model which apply to ideal, random walk chains. Eq. (3.13) is consistent with the dumbbell expressions (3.8)-(3.11) in $d=2$ for $\alpha=0, x_{\epsilon}$ arbitrary, and the Gaussian expressions contained in (3.15) and (3.14) with their two-dimensional counterparts (3.9) and (3.10), (3.11) with $x_{\epsilon}=0$. 


\section{Weakly anisotropic particles}

Here we generalize previous results ${ }^{5}$ for the amplitudes $\mathcal{I}, \mathcal{I}^{\prime}, \mathcal{N}, \mathcal{N}^{\prime}$ of weakly anisotropic particles interacting with ideal chains to the case of self-avoiding chains in a good solvent. As in Ref. 5 we consider particles with a surface $S^{\prime}$ that is obtained by shifting each surface point $\mathbf{r}_{S}$ of the sphere $S$ with radius $R$ by a small amount $\eta\left(\theta_{S}\right)$ toward the center of $S$ at the origin. Here $\theta_{S}$ is the angle between $\mathbf{r}_{S}$ and the symmetry axis of the particle, and we consider particles with a center of reflection, so that $\eta\left(\theta_{S}\right)=\eta\left(\pi-\theta_{S}\right)$. The shift can be generated by means of the stress tensor using correlation functions that are given in Appendix B. This yields

$$
\begin{gathered}
\mathcal{I}=(2 R)^{x_{\epsilon}}\left[1-x_{\epsilon} J\right], \\
\mathcal{I}^{\prime}=\frac{1 / 8}{2\left(x_{\epsilon}+1\right)-d}(2 R)^{x_{\epsilon}+2}\left[1-\left(x_{\epsilon}+2\right) J\right], \\
\mathcal{N}^{\prime}=-(2 R)^{x_{\epsilon}+2} \frac{(d+1) \Gamma((d+2) / 2)}{8 \sqrt{\pi}\left(x_{\epsilon}+1\right) \Gamma((d+1) / 2)} I,
\end{gathered}
$$

and

$$
\mathcal{N}=-R^{d} \frac{\beta_{\text {surf }}}{\beta_{\text {bulk }}} \frac{\pi^{(d-1) / 2}}{\Gamma((d+1) / 2)} I,
$$

where

$$
\begin{gathered}
J=\frac{\Gamma(d / 2)}{\sqrt{\pi} \Gamma((d-1) / 2)} \int_{0}^{\pi} \mathrm{d} \theta_{S}\left(\sin \theta_{S}\right)^{d-2} \eta\left(\theta_{S}\right) / R \\
I=\int_{0}^{\pi} \mathrm{d} \theta_{S}\left(\sin \theta_{S}\right)^{d-2}\left[d\left(\cos \theta_{S}\right)^{2}-1\right] \eta\left(\theta_{S}\right) / R .
\end{gathered}
$$

Here $\beta_{\text {surf }} / \beta_{\text {bulk }}$ is the amplitude ratio of the stress-tensor two-point functions with both points in the planar surface of the half space and in the bulk, respectively. See the discussion pertaining to Eq. (B4). For $d=4-\varepsilon$,

$$
\frac{\beta_{\text {surf }}}{\beta_{\text {bulk }}}=2\left[1-\frac{5}{6} \kappa \varepsilon+O\left(\varepsilon^{2}\right)\right]
$$

depends on $N$, see Ref. 24, while in $d=2$,

$$
\frac{\beta_{\text {surf }}}{\beta_{\text {bulk }}}=2
$$


independent of $N$. The integral $I$ in (3.22) is positive (negative) for oblate (prolate) shapes.

Eqs. (3.17)-(3.24) may be used, in particular, for ellipsoids, lenses, and dumbbells. Weakly anisotropic prolate and oblate ellipsoids with $D_{\|}>D_{\perp}$ and $D_{\|}<D_{\perp}$, respectively, are described ${ }^{5}$ by $R=D_{\|} / 2$ and

$$
\eta=\frac{D_{\|}-D_{\perp}}{2} \sin ^{2} \theta_{S},
$$

to first order in $D_{\|}-D_{\perp}$, and a dumbbell and lens with $\delta \equiv \alpha-\pi<0$ and $>0$, respectively, by $R=L / 2$ and

$$
\eta=\delta(L / 4)\left|\cos \theta_{S}\right|
$$

to first order in $\delta$. Substituting in Eqs. (3.17)-(3.24) yields

$$
\begin{gathered}
\mathcal{I}=\left\{D_{\|}^{x_{\epsilon}}\left[1+\hat{g} x_{\epsilon} \frac{d-1}{d}\right], L^{x_{\epsilon}}\left[1-\delta x_{\epsilon} \omega\right]\right\}, \\
\mathcal{I}^{\prime}=\frac{1 / 8}{2\left(x_{\epsilon}+1\right)-d}\left\{D_{\|}^{x_{\epsilon}+2}\left[1+\hat{g}\left(x_{\epsilon}+2\right) \frac{d-1}{d}\right], L^{x_{\epsilon}+2}\left[1-\delta\left(x_{\epsilon}+2\right) \omega\right]\right\}, \\
\mathcal{N}^{\prime}=\frac{-1}{x_{\epsilon}+1}\left\{D_{\|}^{x_{\epsilon}+2} \hat{g} \frac{d+1}{4(d+2)}, L^{x_{\epsilon}+2} \delta \frac{\omega d}{8}\right\}, \\
\mathcal{N}=-\frac{\beta_{\text {surf }}}{\beta_{\text {bulk }}}\left\{D_{\|}^{d} \hat{g} \frac{(\pi / 4)^{d / 2}}{\Gamma((d+4) / 2)},\left(\frac{L}{2}\right)^{d} \delta \frac{\pi^{(d-1) / 2}}{2 \Gamma((d+3) / 2)}\right\},
\end{gathered}
$$

for the ellipsoid and lens/dumbbell, where

$$
\hat{g}=\left(D_{\perp}-D_{\|}\right) / D_{\|}, \omega=\frac{\Gamma(d / 2)}{2 \sqrt{\pi} \Gamma((d+1) / 2)} .
$$

For the weakly anisotropic ellipse, lens, and dumbbell in $d=2$, Eqs. (3.27)-(3.30) are consistent with (3.5)-(3.11). For the weakly anisotropic ellipsoid in arbitrary $d$ with mean diameter $\mathcal{C}=\left[D_{\|}+(d-1) D_{\perp}\right] / d$, Eqs. (3.27) and (3.28) are equivalent to $\mathcal{I}=\mathcal{C}^{x_{\epsilon}}$ and $\mathcal{I}^{\prime}=\mathcal{C}^{x_{\epsilon}+2} /\left\{8\left[2\left(x_{\epsilon}+1\right)-d\right]\right\}$, apart from corrections of order $\hat{g}^{2}$. For $N=-2$ where $\kappa=0, \beta_{\text {surf }} / \beta_{\text {bulk }}=2, x_{\epsilon}=d-2$ the above expressions reduce to the ideal chain results given in Sec. 5 of Ref. 5 .

Note the $N$ dependence of the leading anisotropy amplitude $\mathcal{N}$ given by Eqs. (3.6), (3.9), (3.15), (3.20), and (3.30). While $\mathcal{N}$ is independent of $N$ for $d=2$ and $d=4, \mathcal{N}$ depends on $N$ for $2<d<4$, and has a smaller magnitude for self-avoiding chains with $N=0, \kappa=1 / 4$ than for ideal, random-walk, chains with $N=-2, \kappa=0$. 


\section{EFFECTIVE INTERACTION BETWEEN A PARTICLE AND A WALL}

The free energy cost of immersing a particle in the polymer solution in the half space bounded by a planar wall follows from Eq. (2.7) on identifying the Hamiltonian $\mathcal{H}$ in the absence of the particle with the Hamiltonian $\mathcal{H}_{\mathrm{hs}}$ of the field theory in the half space and on substituting the Boltzmann weight (2.1) of the particle. For the leading isotropic and anisotropic contributions of the polymer-induced particle-wall interaction this yields

$$
\delta F_{\text {iso }}(\zeta)=p_{0}\left(\mathcal{I} / 2^{x_{\epsilon}}\right) A \mathcal{R}_{x}^{1 / \nu}\left(\mathcal{M}_{\mathrm{hs}}-1\right)
$$

and

$$
\delta F_{\text {aniso }}(\vartheta, \zeta)=\frac{p_{0} \mathcal{N}}{d-1}(\cos \vartheta)^{2}\left\{d\left(1-\mathcal{E}_{\mathrm{hs}}\right)+(\gamma / \nu)\left(\mathcal{M}_{\mathrm{hs}}-\mathcal{E}_{\mathrm{hs}}\right)-\zeta \frac{\mathrm{d}}{\mathrm{d} \zeta} \mathcal{M}_{\mathrm{hs}}\right\}
$$

as shown at the end of Sec. II and in Appendix C, respectively. Here $\delta F_{\text {iso }}(\zeta)=F_{\text {iso }}(\zeta)-$ $F_{\text {iso }}(\infty)$ is the free energy change on moving the particle center from the bulk to a finite distance $z$ from the wall, with

$$
\zeta=z / \mathcal{R}_{x}
$$

and $\delta F_{\text {aniso }}(\vartheta, \zeta)=F_{\text {aniso }}(\vartheta, \zeta)-F_{\text {aniso }}(\pi / 2, \zeta)$ on turning the particle axis, at fixed $z$, from an orientation parallel to the wall to an orientation that encloses an angle $\vartheta$ with the surface normal of the wall. $\mathcal{E}_{\mathrm{hs}}=\mathcal{E}_{\mathrm{hs}}(\zeta)$ and $\mathcal{M}_{\mathrm{hs}}=\mathcal{M}_{\mathrm{hs}}(\zeta)$ are the bulk-normalized density profiles of the chain ends and of all the chain-monomers, respectively, in the dilute polymer solution in the half space without a particle. In terms of the field theory,

$$
\mathcal{E}_{\mathrm{hs}}\left(\zeta_{2}\right)=\Xi_{\mathrm{hs}} / \Xi_{\text {bulk }}
$$

where

$$
\Xi_{\mathrm{hs}}=\int \mathrm{d} \mathbf{r}_{1} \mathcal{L}\left\langle\varphi_{12}\right\rangle_{\mathrm{hs}}, \Xi_{\text {bulk }}=\int \mathrm{d} \mathbf{r}_{1} \mathcal{L}\left\langle\varphi_{12}\right\rangle_{\text {bulk }}
$$

and $\mathcal{M}_{\mathrm{hs}}$ is given by Eq. (2.9). The exponents and amplitudes in (4.1), (4.2), and in (4.6) below are those of the $N \rightarrow 0$ vector model. The exponent $\gamma$ also appears in the power-law dependence $M^{\gamma-1}$ of the partition function $\Xi_{\text {bulk }} / N$ of a polymer chain in the bulk with one end fixed.

The corresponding anisotropic contribution

$$
\delta F_{\text {aniso }}^{(\text {next })}(\vartheta, \zeta)=p_{0}\left(\mathcal{N}^{\prime} / 2^{x_{\epsilon}}\right) A(\cos \vartheta)^{2} \mathcal{R}_{x}^{(1 / \nu)-2} \mathrm{~d}^{2} \mathcal{M}_{\mathrm{hs}} / \mathrm{d} \zeta^{2}
$$


from $\mathcal{N}^{\prime}$ is a next-to-leading correction, since for self-avoiding chains and a small particle, $\mathcal{N}^{\prime}$ is much smaller than $\mathcal{N}$, as explained in the Introduction. Eqs. (4.1), (4.2), and (4.6) apply to particle sizes much smaller than $z$ and $\mathcal{R}_{x}$.

It is interesting to compare the expressions in Eqs. (4.1), (4.2), (4.6) for self-avoiding chains with the ideal-chain expressions ${ }^{5,26}$, in which the two anisotropic contributions from $\mathcal{N}^{\prime}$ and $\mathcal{N}$ in (4.2) and (4.6) are of the same order (size) $)^{d}$. In the latter case

$$
\begin{aligned}
\mathcal{E}_{\mathrm{hs}} & \rightarrow 1-f_{0}(y / 2), \\
\mathcal{M}_{\mathrm{hs}} & \rightarrow 1+4\left[f_{2}(y)-2 f_{2}(y / 2)\right], \\
\mathrm{d}^{2} \mathcal{M}_{\mathrm{hs}} / \mathrm{d} \zeta^{2} & \rightarrow 8\left[f_{0}(y)-(1 / 2) f_{0}(y / 2)\right],
\end{aligned}
$$

independent of $d$, the curly bracket in Eq. (4.2) becomes

$$
\{\} \rightarrow 2 f_{0}(y)+(d-2) f_{0}(y / 2),
$$

and the sphere ${ }^{10}$ amplitude $A \rightarrow A_{\text {ideal }}=2 \pi^{d / 2} / \Gamma((d / 2)-1)$. In Eqs. (4.7) and (4.8), $f_{n}(y)=\mathrm{i}^{n} \operatorname{erfc}(y)$ is the $n$-fold iterated complementary error function of $y=z / \sqrt{L_{\text {pol }}}=\sqrt{2} \zeta$. For ideal chains in $d=3$ the sum of the right hand sides of (4.2) and (4.6) reduces ${ }^{13}$ to the expression given on the right hand side of Eq. (6.1) of Ref. 5.

First consider the region $\zeta \ll 1$ of small particle-wall distances, where the form

$$
\delta F_{\text {aniso }}=p_{0}(\cos \vartheta)^{2}\left\{\mathcal{N} \frac{d}{d-1}\right\}
$$

of the leading anisotropic interaction for self-avoiding chains implies that the particle aligns parallel to the wall. The reason is that $\mathcal{N}$ is positive (negative) for particles that are stretched out parallel (perpendicular) to the particle axis, see Sec. III, so that $\delta F_{\text {aniso }}$ is minimized for $\vartheta=\pi / 2(\vartheta=0)$.

Eq. (4.9) should be compared with the interaction

$$
\delta F_{\text {aniso, ideal }}=p_{0}(\cos \vartheta)^{2}\left\{\mathcal{N} \frac{d}{d-1}+\left(\mathcal{N}^{\prime} / 2^{x_{\epsilon}}\right) 4 A\right\}_{\text {ideal }}
$$

for ideal chains, corresponding to the sum of (4.2) and (4.6). In the last paragraph of Sec. IIIC we argued ${ }^{27}$ that $0<\mathcal{N} / \mathcal{N}_{\text {ideal }}<1$ for the dumbbell/lens and ellipsoid families in $2<d<4$. Since both terms in \{\}$_{\text {ideal }}$ have the same $\operatorname{sign}^{5}$, the ratio \{\}$/\{\}_{\text {ideal }}$ of the curly brackets in (4.9) and (4.10) is also between 0 and 1, and the tendency of the particle to 
align parallel to the wall is reduced by chain self-avoidance. Note that the chain size $\mathcal{R}_{x}$ does not $^{28}$ appear in Eqs. (4.9) and (4.10).

Second we discuss whether the orientation parallel to the wall remains favorable for larger particle-wall scaled distances $\zeta$ of order 1 or much larger than 1 . For ideal chains this is not the case $e^{5,7}$. For large $\zeta$, the anisotropic interaction $\delta F_{\text {aniso, ideal }}(\vartheta, \zeta)$ equals the

product of $\sqrt{2 / \pi} \zeta^{-1} \exp \left(-\zeta^{2} / 2\right)$, which is the large $\zeta$ behavior of $f_{0}(y / 2)$, and the factor $p_{0}(\cos \vartheta)^{2} \mathcal{N}_{\text {ideal }}[(d-2) /(d-1)] k$, where

$$
k=1-\left(\mathcal{N}^{\prime} / \mathcal{N}\right)_{\text {ideal }} 2^{4-d} \pi^{d / 2}(d-1) / \Gamma(d / 2) .
$$

For large $\zeta$ and $d>2, \delta F_{\text {aniso, ideal }}$ is minimized if the particle aligns perpendicular to the wall, since $k$ is negative for all the particle shapes considered. For example, $k$ equals $2-2^{d}$ for a dumbbell of two touching spheres, and equals $1-[d(d+1) / 2]$ for weakly anisotropic particles of the general shape considered in Sec. IIIC.

The situation is different for self-avoiding chains in $d<4$, for which the $\mathcal{N}$ contribution (4.2) dominates the anisotropic behavior of a small particle, and the $\mathcal{N}^{\prime}$ contribution (4.6) is negligible. For $d=4-\varepsilon$ with small positive $\varepsilon$, the value of the curly bracket in (4.2) must be close to its $d=4$ expression, i.e. to the ideal-chain expression $2\left[f_{0}(y)+f_{0}(y / 2)\right]$ of $(4.8)$, which is positive for all values of $y$. This suggests ${ }^{29}$ that the favorable particle orientation is parallel to the wall for all $\zeta$.

\section{SUMMARY AND CONCLUDING REMARKS}

In this paper we consider the orientation-dependent effective interaction between nonspherical colloidal particles immersed in a solution of nonadsorbing polymers. With methods of field theory we study how the interaction is affected by the repulsion between chain monomers (chain self-avoidance) in a good solvent. It is interesting to compare different particle shapes, and we consider ellipsoids, dumbbells, and lenses.

For small particle size we represent the particle by isotropic and anisotropic operators with weights or amplitudes that depend on the particle size and shape and have different values for the universality classes of ideal (random walk) and self-avoiding polymer chains. Nonadsorbing polymers correspond to the surface-universality-class of the 'ordinary transition'. 
Here is a summary of the main results:

1. For self-avoiding polymers in two dimensions the operator weights are evaluated exactly for particles with the shapes of an ellipse of arbitrary aspect ratio and for a dumbbell and a lens of arbitrary angle of intersection of the two circular surfaces. The results are given in Eqs. (3.5)-(3.11). In Figs. 1 and 2 the leading anisotropic weight $\mathcal{N}$ of a lens or dumbbell is compared with that of the smallest circumscribing ellipse. As expected the polymers sense the lens or dumbbell as less or more anisotropic than the ellipse. In Appendix A we confirm the operator form of the expansion by checking that the one- and two-point functions around the particle are described by the same weights. Unlike the operator-algebra in the bulk, the leading anisotropic amplitude $\mathcal{N}$ of the stress tensor does not depend on the bulk universality class, i.e. $\mathcal{N}$ is 'hyper-universal' and independent of the number $N$ of components of the order parameter in the $N$-vector model. As expected from the structure of the operator algebra in the bulk, in the small particle expansion in $d=2$ there is another anisotropic operator besides $\partial_{\|}^{2} \psi$, of scaling dimension $x_{\epsilon}+2$, which is given in Eq. (3.1). The ratio $\mathcal{N}^{\prime} / \mathcal{N}^{\prime \prime}$ of the weights of the two operators is the same for the ellipse, dumbbell, and lens and is independent of their aspect ratios.

2. In more than two spatial dimensions we consider a dumbbell of two touching spheres and weakly anisotropic particles (weakly deformed spheres). Unlike the situation in $d=2$, in $2<d<4$ the leading anisotropic particle-weight $\mathcal{N}$ depends on $N$. For a given particle size and shape in $2<d<4$, both the isotropic and anisotropic perturbation strengths $\mathcal{I} A$ and $\mathcal{N}$ of the polymer system are weaker for self-avoiding than for ideal chains. In the case of $\mathcal{I} A$ this is due to a larger size exponent $d-1 / \nu>d-2$. In the case of $\mathcal{N}$ the size exponent, which equals $d$, is the same but the amplitude is weaker, see Eqs. (3.15), (3.20), (3.30) and the last paragraph in Sec. III. The weights for weakly anisotropic particles are fully determined by the scaling dimension $x_{\epsilon}$ of the energy density and the ratio $\beta_{\text {surf }} / \beta_{\text {bulk }}$ of the amplitudes of the stress-tensor two-point function in the bulk and at the planar surface of the half space, as shown in Appendix B.

3. Using the small-particle expansion, we express the polymer-induced orientationdependent interaction between a particle and a wall in terms of polymer density profiles in the half space bounded by the wall and without particle. For the leading orientation dependence this follows from the continuity and trace equations of the stress tensor, as shown in Appendix C. Apart from reducing the orientation-dependent interaction at particle-wall 
distances $z$ much smaller than the chain size, see Eqs. (4.9), (4.10), chain self-avoidance also affects the qualitative form of its $z$-dependence. Unlike ideal chains, which induce a change in particle-orientation from parallel to perpendicular to the wall on increasing $z$, self-avoiding chains induce a parallel orientation for all distances, as we argue in the last two paragraphs of Sec. IV.

\section{Acknowledgments}

It is a pleasure to thank T.W. Burkhardt for his interest and useful discussions.

\section{APPENDIX A: ELLIPSES, LENSES, AND DUMBBELLS}

\section{IN TWO DIMENSIONS}

Here we discuss density profiles and two-point functions in two-dimensional conformal field theories with a single embedded ellipse, lens, or dumbbell and show that they imply the small particle amplitudes given in Section IIIA.

For later reference we note the relations

$$
\begin{gathered}
\Delta=4 \partial_{w} \partial_{\bar{w}}=4 L_{-1} \bar{L}_{-1}, \\
\partial_{\|}^{2}-\frac{1}{2} \Delta=\rho_{+}^{2} L_{-1}^{2}+\rho_{-}^{2} \bar{L}_{-1}^{2}, \\
T_{\|\|}=-\left(\rho_{+}^{2} T+\rho_{-}^{2} \bar{T}\right) /(2 \pi),
\end{gathered}
$$

with the complex notation introduced below Eq. (3.4).

It is remarkable that the contributions $\propto L_{-1}^{2} \psi, \bar{L}_{-1}^{2} \psi$ cancel in the anisotropic Boltzmann weight reduction

$$
\mathcal{N}^{\prime}\left[\partial_{\|}^{2}-\frac{1}{2} \Delta\right] \psi+\mathcal{N}^{\prime \prime} \tilde{\psi}=-\mathcal{N}^{\prime \prime}\left[\rho_{+}^{2} L_{-2}+\rho_{-}^{2} \bar{L}_{-2}\right] \psi
$$

of order (size $)^{x_{\epsilon}+2}$ in Eqs. (2.3) and (3.2). This follows from (A2) and the forms (3.11) and (3.1), (3.4) of $\mathcal{N}^{\prime} / \mathcal{N}^{\prime \prime}$ and $\tilde{\psi}$.

In the following we assume that the particle is located at the origin with its axis aligned along the $y$-direction of the $x, y$ plane, i.e. $\rho_{+}^{2}=\rho_{-}^{2}=-1$. 


\section{Density profiles}

First consider density profiles of primary operators $\mathcal{O}$, such as the energy density $\mathcal{O}=\epsilon$, that are induced by a single particle. For an ellipse (ell)

$$
\langle\mathcal{O}(w, \bar{w})\rangle_{\mathrm{ell}} / B_{\mathcal{O}}^{1 / 2}=\mathcal{A}_{\mathcal{O}}(2 \mathcal{C})^{x_{\mathcal{O}}}\left\{\frac{|1+(w / \mathcal{S})|}{|w+\mathcal{S}|^{2}-\mathcal{C}^{2}}\right\}^{x_{\mathcal{O}}}
$$

with

$$
\mathcal{S}=\sqrt{w^{2}-\mathcal{C}^{2} g}
$$

and for a dumbbell or lens $(\mathrm{dbl})$

$$
\begin{aligned}
& \langle\mathcal{O}(w, \bar{w})\rangle_{\mathrm{dbl}} / B_{\mathcal{O}}^{1 / 2}=\mathcal{A}_{\mathcal{O}}\left(\frac{\mathcal{D} \pi}{\alpha}\right)^{x_{\mathcal{O}}}\left\{\left|w^{2}-(\mathcal{D} / 2)^{2}\right|\right. \\
& \left.\times \operatorname{Re}\left[\left(\frac{(w+(\mathcal{D} / 2))(\bar{w}-(\mathcal{D} / 2))}{(\bar{w}+(\mathcal{D} / 2))(w-(\mathcal{D} / 2))}\right)^{\pi /(2 \alpha)}\right]\right\}^{-x_{\mathcal{O}}}
\end{aligned}
$$

where 'Re' denotes the real part.

Next consider the stress tensor profiles. Using the complex notation ${ }^{19}$ introduced below Eq. (3.4), one finds

$$
\langle T(w)\rangle_{\mathrm{ell}}=\frac{c}{8} \frac{\mathcal{C}^{2} g}{\mathcal{S}^{4}}
$$

and

$$
\langle T(w)\rangle_{\mathrm{dbl}}=\frac{c}{24}\left[1-\left(\frac{\pi}{\alpha}\right)^{2}\right] \frac{\mathcal{D}^{2}}{\left[w^{2}-(\mathcal{D} / 2)^{2}\right]^{2}},
$$

where $c$ is the central charge of the two-dimensional theory.

Conformal transformations of the two-point function $\langle T \mathcal{O}\rangle$ from the half plane to the exterior of the particle allow one to evaluate the profile of the operator $L_{-2} \mathcal{O}$ around the particle. For the particle families $\{$ ell, dbl $\}$ this leads to

$$
\langle\Lambda \mathcal{O}(w, \bar{w})\rangle_{\mathrm{part}}=\langle\mathcal{O}(w, \bar{w})\rangle_{\mathrm{part}} \lambda\left\{\frac{\mathcal{C}^{2} g}{8 \mathcal{S}^{4}}, \frac{\mathcal{D}^{2}}{\left[w^{2}-(\mathcal{D} / 2)^{2}\right]}\left[1-\left(\frac{\pi}{\alpha}\right)^{2}\right] / 24\right\},
$$

with $\Lambda \mathcal{O}$ from (3.4), and

$$
\lambda \equiv c+x_{\mathcal{O}}\left(4-\frac{9}{x_{\mathcal{O}}+1}\right)
$$


One may check that the above ellipse- and dumbbell/lens-profiles coincide for the circle and the needle, compare the paragraph below Eq. (3.11).

The results (3.5)-(3.11) for the small particle amplitudes follow from comparing the profiles (A5)-(A10) for particle size much smaller than $|w|$ with the form predicted by the expansion (2.1)-(2.3). For example, the result (3.5) for the ellipse-amplitude $\mathcal{I}$ follows from comparing the leading behavior $\mathcal{A}_{\epsilon} \mathcal{C}^{x_{\epsilon}}|w|^{-2 x_{\epsilon}}$ of (A5) for $\mathcal{O}=\epsilon$ and $\mathcal{C} \ll|w|$ with the prediction $\mathcal{I} \mathcal{A}_{\epsilon}\langle\epsilon(0,0) \epsilon(w, \bar{w})\rangle_{\text {bulk }} / B_{\epsilon}=\mathcal{I} \mathcal{A}_{\epsilon}|w|^{-2 x_{\epsilon}}$ from (2.1) and (2.2) and taking the relation (2.4) between $\epsilon$ and $\psi$ into account. Due to the form

$$
\left\langle(\Lambda \mathcal{O}(w, \bar{w}))\left(\Lambda \mathcal{O}\left(w_{\mathrm{P}}, \bar{w}_{\mathrm{P}}\right)\right)\right\rangle_{\text {bulk }} / B_{\mathcal{O}}=\frac{1}{\left|w-w_{\mathrm{P}}\right|^{2 x_{\mathcal{O}}}} \frac{1}{\left(w-w_{\mathrm{P}}\right)^{4}} \lambda / 2
$$

of the bulk two-point function of $\Lambda \mathcal{O}$ and the vanishing mixed function $\langle(\Lambda \mathcal{O})(\bar{\Lambda} \mathcal{O})\rangle_{\text {bulk }}$, the coefficient $\lambda$ drops out of the particle amplitude $\mathcal{N}^{\prime \prime}$. Similarly $c$ drops out of $\mathcal{N}$.

In the next subsection we check the operator character of the expansion by showing that the same amplitudes (3.5)-(3.11) appear also in the small-particle expansion of the two-point function $\langle\epsilon \epsilon\rangle_{\text {part }}$.

\section{Energy-density two-point function in the $N$-vector model}

In the following we use the notation

$$
\mathcal{O}\left(w_{j}, \bar{w}_{j}\right)=\mathcal{O}(j) ; j=1,2
$$

and we omit the spatial arguments of operators that are located at the particle site at the origin. The form

$$
\langle\mathcal{O}(1) \mathcal{O}(2)\rangle_{\text {part }} /\left[\langle\mathcal{O}(1)\rangle_{\text {part }}\langle\mathcal{O}(2)\rangle_{\text {part }}\right]=\mathcal{F}(q) /\left[\mathcal{A}_{\mathcal{O}} 2^{x_{\mathcal{O}}}\right]^{2}
$$

of the ratio of the two-point function and the profiles of a primary operator $\mathcal{O}$ applies not only to the half space problem $z \equiv r_{y}>0$, where the 'particle' fills the complementary half space $z<0$, but also to our particles with ellipse- and dumbbell/lens-shapes. For a given bulk universality class, operator $\mathcal{O}$, and 'ordinary' boundary conditions on the particle surfaces $^{11,12}$, the three cases are described by the same amplitude $\mathcal{A}_{\mathcal{O}}$ and the same function $\mathcal{F}(q)$, but the arguments $q$ are different. For the half space

$$
q=\frac{r_{12}^{2}}{4 z_{1} z_{2}}=-\frac{\left|w_{12}\right|^{2}}{\left(w_{1}-\bar{w}_{1}\right)\left(w_{2}-\bar{w}_{2}\right)},
$$


for the ellipse

$$
q=\mathcal{C}^{2} \frac{\left|w_{1}+\mathcal{S}_{1}-w_{2}-\mathcal{S}_{2}\right|^{2}}{\left(\left|w_{1}+\mathcal{S}_{1}\right|^{2}-\mathcal{C}^{2}\right)\left(\left|w_{2}+\mathcal{S}_{2}\right|^{2}-\mathcal{C}^{2}\right)},
$$

and for the dumbbell/lens particles

$$
q=|G|^{2} /\left(c_{1} c_{2}\right)
$$

with

$$
\begin{aligned}
G & =\left(\theta_{+, 1} \theta_{-, 2}\right)^{\pi / \alpha}-\left(\theta_{-, 1} \theta_{+, 2}\right)^{\pi / \alpha}, \\
c_{i} & =2 \operatorname{Re}\left(\theta_{+, i} \bar{\theta}_{-, i}\right)^{\pi / \alpha},
\end{aligned}
$$

where

$$
\begin{gathered}
\theta_{+, i}=1+\left[\mathcal{D} /\left(2 w_{i}\right)\right], \theta_{-, i}=1-\left[\mathcal{D} /\left(2 w_{i}\right)\right], \\
\bar{\theta}_{+, i}=1+\left[\mathcal{D} /\left(2 \bar{w}_{i}\right)\right], \bar{\theta}_{-, i}=1-\left[\mathcal{D} /\left(2 \bar{w}_{i}\right)\right],
\end{gathered}
$$

and $i=1,2$.

For the energy density $\mathcal{O}=\epsilon$ in the $N$-vector model, the function $\mathcal{F}(q)$ has been evaluated in Ref. 21. For $q$ not too large it has the form

$$
\mathcal{F}=\sum_{i=1}^{3} B_{i} h_{i}^{<}(q(1+q))
$$

where

$$
\begin{aligned}
& h_{1}^{<}(\xi)=\xi^{-2 \Delta} F(-2 \Delta,-3 \Delta / 2,(1-\Delta) / 2 ;-3 \Delta, 1-\Delta ;-4 \xi), \\
& h_{2}^{<}(\xi)=\xi^{-\Delta} F(-\Delta,-\Delta / 2,(1+\Delta) / 2 ;-2 \Delta, 1+\Delta ;-4 \xi) .
\end{aligned}
$$

Here $F={ }_{3} F_{2}$ is a hypergeometric function, $\Delta=x_{\epsilon} / 2$, and

$$
B_{1}=1, B_{2}=2^{x_{\epsilon}} \mathcal{A}_{\epsilon} C_{\epsilon \epsilon \epsilon} / B_{\epsilon}^{3 / 2}
$$

where $C_{\epsilon \epsilon \epsilon}$ is the amplitude of the bulk three-point function $\langle\epsilon(1) \epsilon(2) \epsilon(3)\rangle_{\text {bulk }}=$ $C_{\epsilon \epsilon \epsilon}\left(r_{12} r_{23} r_{31}\right)^{-x_{\epsilon}}$. Eqs. (A24) follow ${ }^{21}$ from comparing the above expressions for $\langle\epsilon(1) \epsilon(2)\rangle_{\mathrm{hs}}$ with the expansion of the operator product $\epsilon(\mathbf{r}-(\mathbf{s} / 2)) \epsilon(\mathbf{r}+(\mathbf{s} / 2))$ for short distance $\mathbf{s}$, as given in Subsection A3 below.

Calculating the contributions of orders $(\text { size })^{x_{\epsilon}},(\text { size })^{2}$, and $(\text { size })^{x_{\epsilon}+2}$ in the small particle expansion of $\langle\epsilon \epsilon\rangle_{\text {part }}$, one can disregard the term with $i=3$ in (A22), which has the small 
$q$ behavior $\propto q^{1+\left(x_{\epsilon} / 2\right)}$. For the ellipse and dumbbell/lens families these contributions follow from (A14), (A22) using the profiles (A5) and (A7), respectively, as well as the expansions in the particle size,

$$
q \rightarrow \frac{\mathcal{C}^{2}}{4} \frac{\left|w_{12}\right|^{2}}{\left|w_{1}\right|^{2}\left|w_{2}\right|^{2}}\left\{1+\frac{\mathcal{C}^{2}}{4}[\mathcal{K}+g \mathcal{J}]+O\left(\mathcal{C}^{4}\right)\right\}
$$

and

$$
q \rightarrow\left(\frac{\pi \mathcal{D}}{2 \alpha}\right)^{2} \frac{\left|w_{12}\right|^{2}}{\left|w_{1}\right|^{2}\left|w_{2}\right|^{2}}\left\{1+\frac{\mathcal{D}^{2}}{4}\left[\left(\frac{\pi}{\alpha}\right)^{2} \mathcal{K}+\frac{1}{3}\left(1-\left(\frac{\pi}{\alpha}\right)^{2}\right) \mathcal{J}\right]+O\left(\mathcal{D}^{4}\right)\right\}
$$

of (A16) and (A17). Here

$$
\mathcal{K}=\frac{1}{\left|w_{1}\right|^{2}}+\frac{1}{\left|w_{2}\right|^{2}}
$$

and

$$
\mathcal{J}=\frac{1}{w_{1}^{2}}+\frac{1}{w_{2}^{2}}+\frac{1}{w_{1} w_{2}}+\mathrm{cc}
$$

are isotropic and anisotropic expressions with 'cc' denoting complex conjugate.

The $i=1$ term in (A22) leads to

$$
\langle\epsilon(1) \epsilon(2)\rangle_{\mathrm{part}}^{(i=1)} / B_{\epsilon}=\left|w_{12}\right|^{-2 x_{\epsilon}}-\mathcal{N}\left\langle T_{\|\|} \epsilon(1) \epsilon(2)\right\rangle_{\mathrm{bulk}} / B_{\epsilon}+O\left((\text { size })^{4}\right)
$$

which contains the contributions of order $(\text { size })^{0}$ and $(\text { size })^{2}$. Here we have used the expansion

$$
h_{1}^{<}(q(1+q))=q^{-x_{\epsilon}}\left(1+O\left(q^{2}\right)\right)
$$

and the form

$$
\left\langle T_{\|\| \|} \epsilon(1) \epsilon(2)\right\rangle_{\text {bulk }} / B_{\epsilon}=\frac{x_{\epsilon}}{4 \pi}\left|w_{12}\right|^{-2 x_{\epsilon}}\left[\left(\frac{1}{w_{1}}-\frac{1}{w_{2}}\right)^{2}+\mathrm{cc}\right]
$$

of the bulk three-point function of $T_{\|\|}$, defined below (2.3), and two energy densities. The same values (3.6), (3.9) of the amplitude $\mathcal{N}$ describe the small-particle behavior of both the profiles (A8), (A9) and the two-point functions (A29). This is consistent with the operator expansion (2.1)-(2.3).

Finally the $i=2$ term in (A22) leads to

$$
\begin{aligned}
& \langle\epsilon(1) \epsilon(2)\rangle_{\text {part }}^{(i=2)} / B_{\epsilon}=\left\{\mathcal{I}\langle\epsilon \epsilon(1) \epsilon(2)\rangle_{\text {bulk }}+\mathcal{I}^{\prime}\langle\Delta \epsilon \epsilon(1) \epsilon(2)\rangle_{\text {bulk }}\right. \\
& \left.+\mathcal{N}^{\prime \prime}\left\langle\left[\left(L_{-2}+\bar{L}_{-2}\right) \epsilon\right] \epsilon(1) \epsilon(2)\right\rangle_{\text {bulk }}\right\} \mathcal{A}_{\epsilon} / B_{\epsilon}^{3 / 2}+O\left((\text { size })^{x_{\epsilon}+3}\right),
\end{aligned}
$$


which contains the contributions of order (size) $)^{x_{\epsilon}}$ and $(\text { size })^{x_{\epsilon}+2}$. Here $\mathcal{I}, \mathcal{I}^{\prime}, \mathcal{N}^{\prime \prime}$ are the amplitudes in (3.5)-(3.11), $\langle\epsilon \epsilon \epsilon\rangle_{\text {bulk }}$ is given below Eq. (A24),

$$
\begin{gathered}
\left\langle\left[\left(L_{-2}+\bar{L}_{-2}\right) \epsilon\right] \epsilon(1) \epsilon(2)\right\rangle_{\text {bulk }} /\langle\epsilon \epsilon(1) \epsilon(2)\rangle_{\text {bulk }}=\frac{x_{\epsilon}}{2}\left[2\left(\frac{1}{w_{1}^{2}}+\frac{1}{w_{2}^{2}}\right)-\frac{1}{w_{1} w_{2}}+c c\right] \\
\langle\Delta \epsilon \epsilon(1) \epsilon(2)\rangle_{\text {bulk }} /\langle\epsilon \epsilon(1) \epsilon(2)\rangle_{\text {bulk }}=x_{\epsilon}^{2}\left|\frac{1}{w_{1}}+\frac{1}{w_{2}}\right|^{2}
\end{gathered}
$$

and we have used

$$
h_{2}^{<}(q(1+q))=q^{-x_{\epsilon} / 2}\left(1-x_{\epsilon} q / 4+O\left(q^{2}\right)\right) \text {. }
$$

It follows from Eqs. (2.2), (2.4), and (A4) that (A32) is consistent with the small-particle operator-expansion.

\section{Particle and operator-product expansions}

Here we compare the small-particle expansion on the right hand side of (2.1) with the expansion of the normalized product $s^{2 x_{\mathcal{O}}} \mathcal{O}(\mathbf{r}-(\mathbf{s} / 2)) \mathcal{O}(\mathbf{r}+(\mathbf{s} / 2)) / B_{\mathcal{O}}$ of two equal primary scalar operators $\mathcal{O}$, such as $\epsilon$, which are separated by the 'small distance' vector s. First, $1-\mathcal{N} \sum_{k l} \rho_{k} \rho_{l} T_{k l}$ with $\mathcal{N}$ in (3.6) and (3.9) should be compared with the contributions ${ }^{9,19}$

$$
1-2 \pi\left(x_{\mathcal{O}} / c\right) \sum_{k l} s_{k} s_{l} T_{k l}
$$

from the unit operator and the stress tensor to the normalized product. Second, the sum of $\left(\mathcal{I}+4 \mathcal{I}^{\prime} L_{-1} \bar{L}_{-1}\right) \psi$ and the right hand side of (A4), when multiplied by -1 , should be compared with the contributions to the normalized product from the operator $\epsilon$ and its descendants, which are given by ${ }^{19}$

$$
\left[C_{\mathcal{O O} \epsilon} /\left(B_{\mathcal{O}} B_{\epsilon}^{1 / 2}\right)\right] s^{x_{\epsilon}}\left\{\mathcal{D}_{0, x_{\epsilon}}\left(\mathbf{s}, \partial_{\mathbf{r}}\right)+f\left[s_{+}^{2} \Lambda+s_{-}^{2} \bar{\Lambda}\right]\right\} \epsilon(\mathbf{r}) / B_{\epsilon}^{1 / 2}
$$

Here $\mathbf{s}=\left(s_{x}, s_{y}\right), s_{ \pm}=s_{x} \pm i s_{y}$,

$$
\mathcal{D}_{0, x_{\epsilon}}\left(\mathbf{s}, \partial_{\mathbf{r}}\right) \epsilon(\mathbf{r})=\left\{1+\frac{1}{8\left(x_{\epsilon}+1\right)}\left(s_{+}^{2} L_{-1}^{2}+s_{-}^{2} \bar{L}_{-1}^{2}\right)\right\} \epsilon(w, \bar{w})
$$

is the same expression as in Eqs. (2.39) and (A1) of Ref. 9, and ${ }^{19}$

$$
f=\frac{x_{\epsilon}\left(x_{\epsilon}-2\right) / 4+x_{\mathcal{O}}\left(x_{\epsilon}+1\right)}{c\left(x_{\epsilon}+1\right)+x_{\epsilon}\left(4 x_{\epsilon}-5\right)} .
$$


There are similarities between the two expansions, with $\vec{\rho}$ times the particle size corresponding to $\mathbf{s}$. The operator product and the particle both have the axial and reflection symmetries and essentially the same operators appear in the two cases. But there are also differences. In the case of the operator product, the amplitude of $T_{k l}$ in (A36) depends on the bulk universality class, while in the particle case the corresponding amplitude $\mathcal{N}$ in (3.6) and (3.9) is 'hyper-universal' (but does depend on the shape of the particle). As for $\epsilon$ and its descendants, the anisotropic operators $\left(L_{-1}^{2}, \bar{L}_{-1}^{2}\right) \epsilon$ are absent in the particle case, see (A4), and the isotropic operator $L_{-1} \bar{L}_{-1} \epsilon$ is absent in the case (A37) of the operator product.

\section{APPENDIX B: SMALL DEFORMATIONS OF A SPHERE}

In field theory, small deformations of a boundary can be generated by means of the stress tensor $^{7,18}$. For the weakly deformed sphere $S^{\prime}$ introduced in Sec. IIIC, the induced profile $\langle\mathcal{O}(\mathbf{r})\rangle_{S^{\prime}}$ of a scalar operator $\mathcal{O}$ such as the energy density $\epsilon$, right at the critical point, is given by

$$
\langle\mathcal{O}(\mathbf{r})\rangle_{S^{\prime}} /\langle\mathcal{O}(\mathbf{r})\rangle_{S}=1-x_{\mathcal{O}}\left(\frac{r^{2}-R^{2}}{R}\right)^{d} \frac{1}{\Omega_{d}} \int \mathrm{d} S \frac{\eta\left(\mathbf{r}_{S} / R\right)}{\left|\mathbf{r}-\mathbf{r}_{S}\right|^{2 d}}+O\left(\eta^{2}\right) .
$$

Here $\Omega_{d}=2 \pi^{d / 2} / \Gamma(d / 2)$ is the surface area of the unit sphere, $\int \mathrm{d} S$ an integral over the surface area of the sphere $S$ with radius $R$ and center at the origin, and the unit vector $\mathbf{r}_{S} / R$ specifies points $\mathbf{r}_{S}$ on the surface of $S$. Eq. (B1) applies to an arbitrary radial surface shift $\eta\left(\mathbf{r}_{S} / R\right)$ and, in particular, to the shift $\eta\left(\mathbf{r}_{S} / R\right) \rightarrow \eta\left(\theta_{S}\right)$ with rotation and reflection symmetry, as in Sec. IIIC. The first order in $\eta$ contribution on the right hand side of (B1) is the explicit form of $\int \mathrm{d} S \eta\left\langle T_{n n}\left(\mathbf{r}_{S}\right) \mathcal{O}(\mathbf{r})\right\rangle_{S} /\langle\mathcal{O}(\mathbf{r})\rangle_{S}$, where $T_{n n}$ is the diagonal component of the stress tensor normal to the surface of $S$. The form of $\left\langle T_{n n} \mathcal{O}\right\rangle_{S} /\langle\mathcal{O}\rangle_{S}$ follows by a special conformal transformation from ${ }^{18}$ the corresponding expression $-x_{\mathcal{O}}\left(2^{d} / \Omega_{d}\right)\left\{z /\left[\left|\mathbf{r}_{p}-\mathbf{r}_{p}^{\prime}\right|^{2}+\right.\right.$ $\left.\left.z^{2}\right]\right\}^{d}$ for $\left\langle T_{n n}\left(\mathbf{r}_{p}^{\prime}, 0\right) \mathcal{O}\left(\mathbf{r}_{p}, z\right)\right\rangle_{\mathrm{hs}} /\left\langle\mathcal{O}\left(\mathbf{r}_{p}, z\right)\right\rangle_{\mathrm{hs}}$ in the half space.

The stress tensor profile is given by

$$
\left\langle T_{k l}(\mathbf{r})\right\rangle_{S^{\prime}}=\beta_{\text {surf }} \int \mathrm{d} S \frac{\eta\left(\mathbf{r}_{S} / R\right)}{\left|\mathbf{r}-\mathbf{r}_{S}\right|^{2 d}}\left\{\delta_{k l}-d v_{k} v_{l}\right\}+O\left(\eta^{2}\right)
$$

with unit vectors

$$
\mathbf{v}=\mathbf{r}_{S} / R-2 \vec{\varrho}\left(\vec{\varrho}, \mathbf{r}_{S} / R\right)
$$


that are composed of the two unit vectors $\mathbf{r}_{S} / R$ and $\vec{\varrho}=\left(\mathbf{r}-\mathbf{r}_{S}\right) /\left|\mathbf{r}-\mathbf{r}_{S}\right|$. This profile vanishes for a sphere and, therefore, for $\eta=0$ where $S^{\prime}=S$. The first order in $\eta$ contribution on the right hand side of (B2) is the explicit form of $\int \mathrm{d} S \eta\left\langle T_{n n}\left(\mathbf{r}_{S}\right) T_{k l}(\mathbf{r})\right\rangle_{S}$. Here $\left\langle T_{n n} T_{k l}\right\rangle_{S}$ follows by a conformal transformation from the half space function ${ }^{24}$

$$
\left\langle T_{n n}(\mathbf{0}) T_{k l}\left(\mathbf{r}_{p}, z\right)\right\rangle_{\mathrm{hs}}=\beta_{\mathrm{surf}} r^{-2 d}\left\{\delta_{k l}-d u_{k} u_{l}\right\}
$$

with the origin $\mathbf{0}$ a surface point and with unit vectors

$$
u_{k}=\delta_{k n}-2 r_{k} z / r^{2}
$$

where $n$ is now the direction perpendicular to the planar surface of the half space, so that $r_{n}=z$.

In the bulk the stress tensor two-point function $\left\langle T_{n n}(\mathbf{0}) T_{k l}(\mathbf{r})\right\rangle_{\text {bulk }}$, with $n$ an arbitrary Cartesian direction, also has the form of the right hand side of (B4), except that $\beta_{\text {surf }}$ is replaced by the bulk amplitude $\beta_{\text {bulk }}$.

Expanding the profiles $\langle\epsilon\rangle_{S^{\prime}}$ and $\left\langle T_{k l}\right\rangle_{S^{\prime}}$ in (B1) and (B2) for $R \ll r$ and comparing with the predictions of the small particle expansion (2.1)-(2.3) leads to Eqs. (3.17)-(3.22). Here one uses the relations

$$
\frac{1}{\Omega_{d}} \int \mathrm{d} S \eta\left(\theta_{S}\right)=R^{d} J
$$

$\int \mathrm{d} S \eta\left(\theta_{S}\right) \cos \left(\mathbf{r}, \mathbf{r}_{S}\right)=0$, and

$$
\frac{1}{\Omega_{d}} \int \mathrm{d} S\left[d \cos ^{2}\left(\mathbf{r}, \mathbf{r}_{S}\right)-1\right] \eta\left(\theta_{S}\right)=R^{d} \frac{\Omega_{d-1}}{\Omega_{d}} \frac{d\left(r_{\|}^{2} / r^{2}\right)-1}{d-1} I,
$$

where $r_{\|}$is the component of $\mathbf{r}$ parallel to the particle axis. The $\eta$ integrals $J$ and $I$ are from (3.21) and (3.22).

\section{APPENDIX C: ENERGY DENSITY, STRESS TENSOR, AND}

\section{PARTICLE-WALL INTERACTION}

First we note general properties of the monomer density operator $\Psi$, which is related to $\epsilon$ by $^{7,10,16,17}$

$$
-\epsilon(\mathbf{r}) \rightarrow\left(L_{\mathrm{pol}} / \mathcal{R}_{x}^{1 / \nu}\right) \Psi(\mathbf{r})
$$


as $N \rightarrow 0$. The 'normalization' property

$$
\mathcal{L} \int \mathrm{d} \mathbf{r}\langle\Psi(\mathbf{r}) \cdot \ldots\rangle=\mathcal{R}_{x}^{1 / \nu} \mathcal{L}\langle\ldots\rangle
$$

follows from the relation $\int \mathrm{d} \mathbf{r}\langle\epsilon(\mathbf{r}) \cdot \ldots\rangle=(\mathrm{d} / \mathrm{d} t)\langle\ldots\rangle$ for the cumulant average, see $(2.6)$, and from partial integration with respect to $t$ inside the Laplace transformation $\mathcal{L}$ in (2.5). Here the dots denote other operators such as $\varphi_{12} / N$. The bulk normalization $\mathcal{M}_{\mathrm{hs}}(\infty)=1$ in Eq. (2.9) is consistent with (C2). The local relation

$$
\mathcal{L}\langle\mathcal{T}(\mathbf{r}) \cdot \ldots\rangle \rightarrow\left(\mathrm{d} / \mathrm{d} \mathcal{R}_{x}^{1 / \nu}\right) \mathcal{L}\langle\Psi(\mathbf{r}) \cdot \ldots\rangle
$$

for $N \rightarrow 0$ follows from replacing the left hand side by $-\left(\mathrm{d} / \mathrm{d} L_{\text {pol }}\right) \mathcal{L}\langle\epsilon(\mathbf{r}) \cdot \ldots\rangle$, see $(2.6)$ and (2.5), and using that $L_{\mathrm{pol}} / \mathcal{R}_{x}^{1 / \nu}$ in (C1) is independent of $L_{\mathrm{pol}}$.

The (naive) inverse length dimension of $\Psi(\mathbf{r})$ equals its scaling dimension $x_{\epsilon}$, and the critical bulk correlation function $\langle\Psi(\mathbf{r}) \Psi(0)\rangle_{\text {bulk }} / N$ for $N \rightarrow 0$ equals the product of $r^{-2 x_{\epsilon}}$ and the universal amplitude

$$
\varpi^{2}=\left(\mathcal{R}_{x}^{1 / \nu} / L_{\text {pol }}\right)^{2}\left(B_{\epsilon} / N\right)_{N=0}
$$

This resembles the universal amplitude $\left(\xi^{1 / \nu} t\right)^{2} B_{\epsilon}$, where $\xi$ is the correlation length, which appears in the critical bulk autocorrelation function of the operator $\xi^{1 / \nu} \mathcal{T}(\mathbf{r})$, where $\mathcal{T}$ is the thermal perturbation density (2.6). By comparing (C1) and (C4) with (2.4) and (2.8), one obtains the expression

$$
A=\left(2^{x_{\epsilon}} \mathcal{A}_{\epsilon} N^{-1 / 2}\right)_{N=0} / \varpi
$$

for the universal polymer amplitude $A$ defined for a sphere in (2.10). In the simple case of ideal polymers and the Gaussian model, $\mathcal{A}_{\epsilon} N^{-1 / 2}=2^{(3 / 2)-d}$ and $B_{\epsilon} / N$ are independent of $N, \varpi=\sqrt{2} \tilde{S}_{d}$ with $\tilde{S}_{d}=\Gamma((d-2) / 2) /\left(4 \pi^{d / 2}\right)$, and $A=A_{\text {ideal }}=1 /\left(2 \tilde{S}_{d}\right)$ is the expression given below Eq. (4.8).

Now we consider the leading anisotropic particle-wall interaction, which by Eqs. (2.1), (2.3), and (2.7) is given by

$$
\delta F_{\text {aniso }}=\frac{p_{0} \mathcal{N}}{d-1}(\cos \vartheta)^{2} \frac{\mathcal{L}\left[d \tau_{n n}-\sum_{l=1}^{d} \tau_{l l}\right]}{\Xi_{\text {bulk }}},
$$

where

$$
\tau_{k l}=\int \mathrm{d} \mathbf{r}_{1} \mathrm{~d} \mathbf{r}_{2}\left\langle T_{k l}(\mathbf{r}) \varphi_{12}\right\rangle_{\mathrm{hs}}
$$


and $\Xi_{\text {bulk }}$ is given in Eq. (4.5). To derive (C6), we used the results that $\tau_{n p}$ vanishes for tensor indices $n$ and $p$ perpendicular and parallel to the wall, respectively, and that $\tau_{n n}-\tau_{p p}$ equals $\left[d \tau_{n n}-\sum_{l} \tau_{l l}\right] /(d-1)$, since the $d-1$ diagonal components $\tau_{p p}$ parallel to the wall are all equal.

The trace $\sum_{l} \tau_{l l}$ and the diagonal component $\tau_{n n}$ perpendicular to the wall follow, respectively, from the trace equation in the scaling limit,

$$
-\sum_{l=1}^{d}\left\langle T_{l l}(\mathbf{r}) \varphi_{12}\right\rangle_{\mathrm{hs}}=x_{\Phi}\left[\delta\left(\mathbf{r}-\mathbf{r}_{1}\right)+\delta\left(\mathbf{r}-\mathbf{r}_{2}\right)\right]\left\langle\varphi_{12}\right\rangle_{\mathrm{hs}}+(1 / \nu)\left\langle\mathcal{T}(\mathbf{r}) \varphi_{12}\right\rangle_{\mathrm{hs}}
$$

with $x_{\Phi}$ the scaling dimension of the order parameter, and the continuity equation,

$$
-\sum_{l} \partial_{r_{l}}\left\langle T_{k l}(\mathbf{r}) \varphi_{12}\right\rangle_{\mathrm{hs}}=\left[\delta\left(\mathbf{r}-\mathbf{r}_{1}\right) \partial_{r_{1 k}}+\delta\left(\mathbf{r}-\mathbf{r}_{2}\right) \partial_{r_{2 k}}\right]\left\langle\varphi_{12}\right\rangle_{\mathrm{hs}}
$$

of the stress tensor ${ }^{30}$. Integrating these equations with respect to $\mathbf{r}_{1}$ and $\mathbf{r}_{2}$ and applying the inverse Laplace transform $\mathcal{L}$, one can express the second fraction on the right hand side of (C6) in terms of the half space profiles $\mathcal{M}_{\mathrm{hs}}$ and $\mathcal{E}_{\mathrm{hs}}$.

From (C3) and (2.9) the contribution from the last term in the trace equation (C8) is

$$
\begin{aligned}
\frac{1}{\nu \Xi_{\text {bulk }}} \int \mathrm{d} \mathbf{r}_{1} \mathrm{~d} \mathbf{r}_{2} \mathcal{L}\left\langle\mathcal{T}(\mathbf{r}) \varphi_{12}\right\rangle_{\mathrm{hs}} & \rightarrow \frac{1}{\nu \Xi_{\text {bulk }}} \frac{\mathrm{d}}{\mathrm{d} \mathcal{R}_{x}^{1 / \nu}}\left(\mathcal{M}_{\mathrm{hs}} \mathcal{R}_{x}^{1 / \nu} \Xi_{\text {bulk }}\right) \\
& \rightarrow-\zeta \mathrm{d} \mathcal{M}_{\mathrm{hs}} / \mathrm{d} \zeta+(\gamma / \nu) \mathcal{M}_{\mathrm{hs}}
\end{aligned}
$$

where the last term comes from differentiating $\mathcal{R}_{x}^{1 / \nu} \Xi_{\text {bulk }}=$ const $\times\left(\mathcal{R}_{x}^{1 / \nu}\right)^{\gamma}$.

With $\Xi_{\mathrm{hs}}$ and $\Xi_{\text {bulk }}$ from (4.5), the expression

$$
\mathcal{L} \tau_{n n}=\Xi_{\text {bulk }}-2 \Xi_{\mathrm{hs}}
$$

follows from the continuity equation (C9), yielding $\partial_{z} \mathcal{L} \tau_{n n}=-2 \partial_{z} \Xi_{\mathrm{hs}}$, and from the bulk value $-\Xi_{\text {bulk }}$ of $\mathcal{L} \tau_{n n}$. The latter can be inferred from the trace equation (C8), since $\mathcal{L} \tau_{n n}$ equals $\mathcal{L} \sum_{l} \tau_{l l} / d$ in the bulk. Here one uses Eq. (C10) for $\zeta \rightarrow \infty$ and the exponent relation $2 x_{\Phi}=d-(\gamma / \nu)$. An alternative derivation of (C11) follows from integrating the shift relation

$$
\int \mathrm{d} \mathbf{r}_{p}\left\langle T_{n n}\left(\mathbf{r}_{p}, z\right) \varphi_{12}\right\rangle_{\mathrm{hs}}=\left[\theta\left(z_{1}-z\right) \partial_{z_{1}}+\theta\left(z_{2}-z\right) \partial_{z_{2}}\right]\left\langle\varphi_{12}\right\rangle_{\mathrm{hs}}
$$

in the half space, where $\theta$ is the standard step function.

Substituting (C8)-(C11) into (C6) and using Eq. (4.4) leads to expression (4.2) for $\delta F_{\text {aniso }}$.

1 Soft Matter, G. Gompper and M. Schick (eds), Wiley-VCH 2005. 
2 D. Rudhardt and C. Bechinger and P. Leiderer, Phys. Rev. Lett. 81, 1330 (1998).

3 A. van Blaaderen, Materials Research Society Bulletin 29, February,85 (2004); P.M. Johnson, C.M. van Kats, and A. van Blaaderen, Langmuir 21, 11510 (2005).

4 Equivalently one may use $\mathcal{R}_{x}=\mathcal{R}_{e} / \sqrt{d}$ or the radius of gyration $\mathcal{R}_{g}$ of the polymer chain. The subscript $x$ in $\mathcal{R}_{x}$ should not be confused with critical exponents $x$.

5 E. Eisenriegler and A. Bringer, J. Phys.: Condens. Matter 17, S1711 (2005).

6 P.G. de Gennes, Scaling Concepts in Polymer Physics (Cornell University, Ithaca, 1979).

7 E. Eisenriegler in: Soft Matter Volume 2, G. Gompper and M. Schick (eds), Wiley-VCH 2005.

8 T. Burkhardt and E. Eisenriegler, Phys. Rev. Lett. 74, 3189 (1995); 78, 2867 (1997).

9 E. Eisenriegler, J. Chem. Phys. 121, 3299 (2004).

10 (a) E. Eisenriegler, Polymers near Surfaces, World Scientific 1993; (b) E. Eisenriegler, in Lecture Notes in Physics, edited by H. Meyer-Ortmanns and A. Klümper, Vol. 508, pp1-24, Springer, Berlin (1998).

11 K. Binder, in Phase Transitions and Critical Phenomena, edited by C. Domb and J. L. Lebowitz (Academic, London, 1983), Vol. 8, p. 1.

12 H. W. Diehl, in Phase Transitions and Critical Phenomena, edited by C. Domb and J. L. Lebowitz (Academic, London, 1986), Vol. 10, p. 75; H. W. Diehl, Int. J. Mod. Phys. B 11, 3503 (1997).

13 Both $\langle\psi(\mathbf{r}) \psi(\mathbf{0})\rangle_{\text {bulk }} r^{2 x_{\epsilon}}$ and $-\left\langle\psi\left(\mathbf{r}_{p}, z\right)\right\rangle_{\mathrm{hs}} z^{x_{\epsilon}}$ equal $\mathcal{A}_{\epsilon}^{2}$. The amplitudes in Eqs. (2.2), (2.3) are related by $\left[\mathcal{I}, \mathcal{I}^{\prime}, \mathcal{N}^{\prime}\right]=\left[a_{1}, a_{2}+\left(b_{1} / d\right), b_{1}\right] B_{\epsilon}^{1 / 2} / \mathcal{A}_{\epsilon}$ and $\mathcal{N}=-b_{2}$ to the amplitudes $a_{1}, a_{2}, b_{1}, b_{2}$ in Refs. 5 and 9. The expression $\mathcal{A}_{\epsilon} / \sqrt{N}=2^{(3 / 2)-d}$ for the Gaussian model in $d$ dimensions ${ }^{9}$ is consistent with the value $\mathcal{A}_{\epsilon}^{2} / N=1 / 2$ for the $N$-vector model in two dimensions ${ }^{20}$ with $N=-2$.

14 The dots in (2.2) and (2.3) also contain terms with integer powers of $t \propto T-T_{c}$ in the prefactors. By scaling-dimensional and locality reasons they must be accompanied by corresponding powers of (particle size $)^{1 / \nu}$. These terms and the $\Delta \psi$ term do not contribute to the leading isotropic and anisotropic behavior.

15 The amplitude $\mathcal{I}$ equals the ratio $\left[\langle\psi(\mathbf{r})\rangle_{\text {particle }}\left|\mathbf{r}-\mathbf{r}_{\mathrm{P}}\right|^{2 x_{\epsilon}}\right] /\left[\left\langle\psi\left(\mathbf{r}^{\prime}\right)\right\rangle_{\mathrm{hs}}\left(z^{\prime}\right)^{x_{\epsilon}}\right]$ for $\left|\mathbf{r}-\mathbf{r}_{\mathrm{P}}\right| \rightarrow \infty$, which is finite for $N \rightarrow 0$.

16 E. Eisenriegler, Phys. Rev. E55, 3116 (1997).

17 A. Hanke, E. Eisenriegler, and S. Dietrich, Phys. Rev. E59, 6853 (1999). 

(Academic Press, London, 1986), Vol. 11, p. 55.

19 A. A. Belavin, A. M. Polyakov, and A. B. Zamolodchikov, Nuclear Phys. B241, 333 (1984).

${ }^{20}$ In $d=2, x_{\epsilon}=2(m-1) /(m+1)$ and $x_{\Phi}=(m+3)(m-1) /[8 m(m+1)]$, where $m$ depends on $N$ via $N=2 \cos (\pi / m)$, so that $1 \leq m \leq \infty$ corresponds to $-2 \leq N \leq 2$. The half space amplitude $\mathcal{A}_{\epsilon}$ is denoted by $2^{-x_{\epsilon}} \mathcal{A}_{O}$ in Ref. 21 and equals $2^{-x_{\epsilon}}\left[-\cos \left(3 \pi x_{\epsilon} / 4\right) / \cos \left(\pi x_{\epsilon} / 4\right)\right]^{1 / 2}$.

21 (a) T.W. Burkhardt, E. Eisenriegler, and I. Guim, Nuclear Phys. B316, 559 (1989); (b) T.W. Burkhardt and E. Eisenriegler, Nuclear Phys. B424, 487 (1994).

22 M. Krech and S. Dietrich, Phys. Rev. A46, 1886 (1992).

23 M. Krech, E. Eisenriegler, and S. Dietrich, Phys. Rev. E52, 1345 (1995).

24 D.M. McAvity and H. Osborn, Nuclear Phys. B406, 655 (1993). In this reference $\beta_{\text {surf }}$ and $\beta_{\text {bulk }}$ are denoted by $\beta(v)$ with $v=1$ and $v=0$, respectively. In Ref. $9,-d \beta_{\text {bulk }}$ is denoted by $B_{T}$.

25 Eqs. (3.12), (3.14) follow via Ref. 13 and Eq. (2.9) in Ref. 9 from the $\varepsilon$-expansion of $\mathcal{E}_{\text {film }}(y) / \mathcal{A}_{\epsilon}=$ $\langle\epsilon\rangle_{\text {film }} /\left(B_{\epsilon}^{1 / 2} \mathcal{A}_{\epsilon}\right)$, which equals $g_{\mathcal{O}, \mathcal{O}}$ in Eq. $(4.16 \mathrm{~b})$ of Ref. 23 with $x$ replaced by $(1+y) / 2$. To first order in $\varepsilon$ the amplitude $B_{T}$ of the bulk two-point function of the stress-tensor in Eqs. (2.15) and (2.17) of Ref. 9 for the $N$-vector model equals $N$ times the amplitude in Eq. (3.35) of Ref. 9 for the Gaussian model, see the discussion below Eq. (3.15) in Ref. 24.

26 The ideal chain profile $\mathcal{M}_{\mathrm{hs}} \equiv \mathcal{M}_{\mathrm{hs}}(\zeta)$ in Eqs. (4.7) equals the quantity $\mathcal{M}_{\mathrm{h}}(y)$ introduced in Ref. 5. Since $y=\sqrt{2} \zeta, \mathcal{M}_{\mathrm{hs}}^{\prime \prime}=2 \mathcal{M}_{\mathrm{h}}^{\prime \prime}$.

27 We only showed $0<\mathcal{N} / \mathcal{N}_{\text {ideal }}<1$ for a dumbbell of two touching spheres and for weakly anisotropic particles, and only to first order in $\varepsilon=4-d$. However, we expect it to apply to the whole dumbbell/lens and ellipsoid families in $d=3$.

28 Eqs. (4.9) and (4.10) should be compared with the isotropic particle-wall interaction (4.1) for small $\zeta$ in which the chain size $\mathcal{R}_{x}$ does appear and which is given, apart from the sign, by the immersion free energy in the bulk, $F_{\text {bulk }}=p_{0}(\text { size })^{d}\left(\mathcal{R}_{x} / \text { size }\right)^{1 / \nu} a$. Here 'size' denotes the particle size and $a=A \mathcal{I} /(2 \times \text { size })^{x_{\epsilon}}$ is the shape-dependent amplitude. $F_{\text {bulk }}$ is also reduced by chain self-avoidance if, in addition to the particle size and shape, $\mathcal{R}_{x}$ is kept fixed. The reason is the decrease of the exponent $1 / \nu$ which is more effective than the change of $a$.

29 It would be interesting to confirm this conjecture by evaluating the half-space profiles $\mathcal{E}_{\text {hs }}$ and $\mathcal{M}_{\text {hs }}$ for self-avoiding chains to first order in $\varepsilon$ (including the one-loop correction) and in $d=2$.

30 L.S. Brown, Ann. Phys. (N.Y.) 126, 135 (1980). 


\section{FIGURE CAPTIONS}

FIG. 1: Small particle amplitude $\mathcal{N}$ which determines the anisotropic interaction of a lens and its circumscribed ellipse CE with self-avoiding polymers in $d=2$ spatial dimensions. The two particle shapes coincide for $\alpha^{\prime}=0$ and $\alpha^{\prime}=\pi$, where they reduce to a needle and a circle, respectively.

FIG. 2: $\mathcal{N}$ for a dumbbell and the circumscribing ellipse. For $\alpha=0$ the dumbbell consists of two touching circles. 


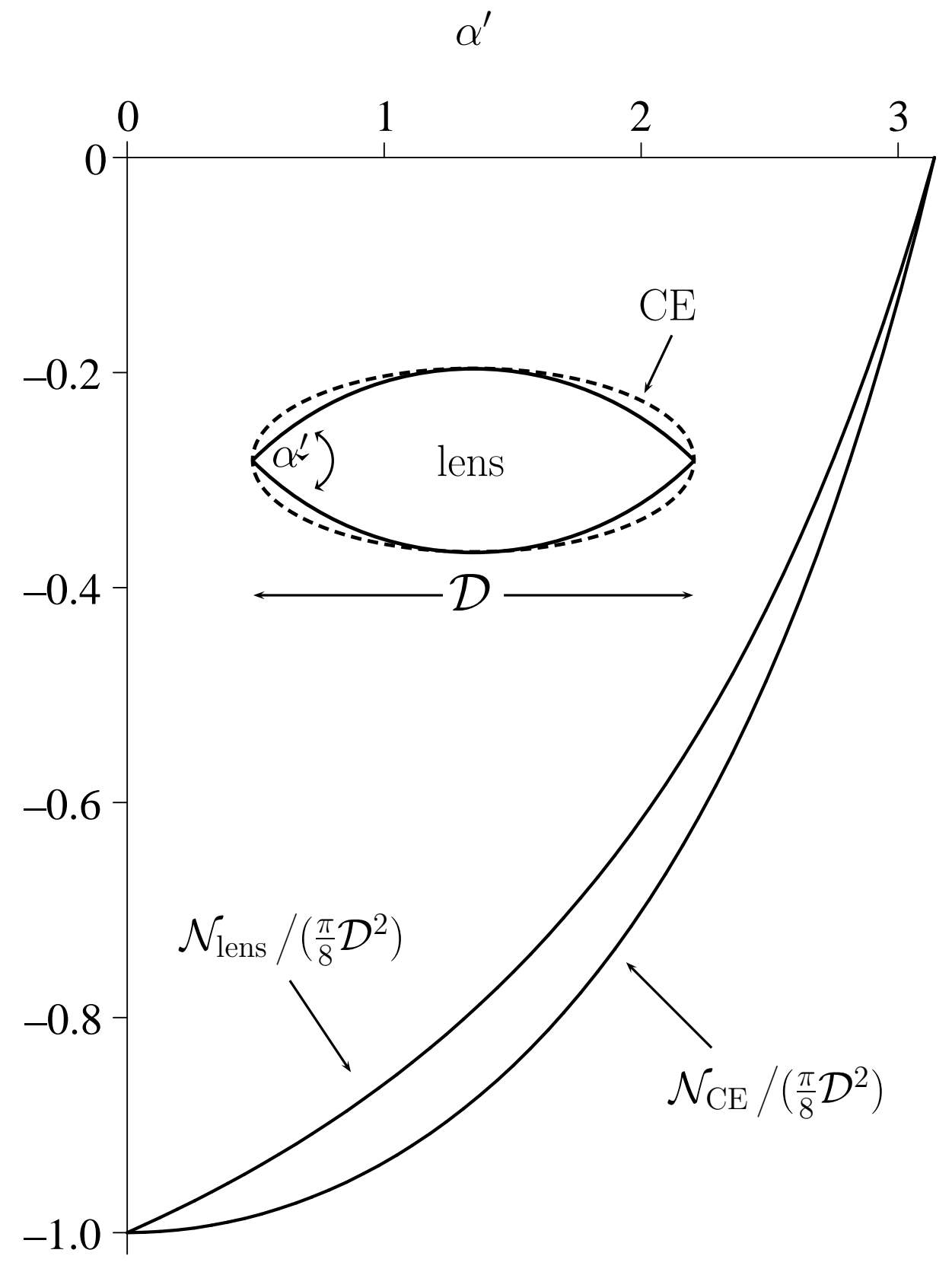

FIG. 1 


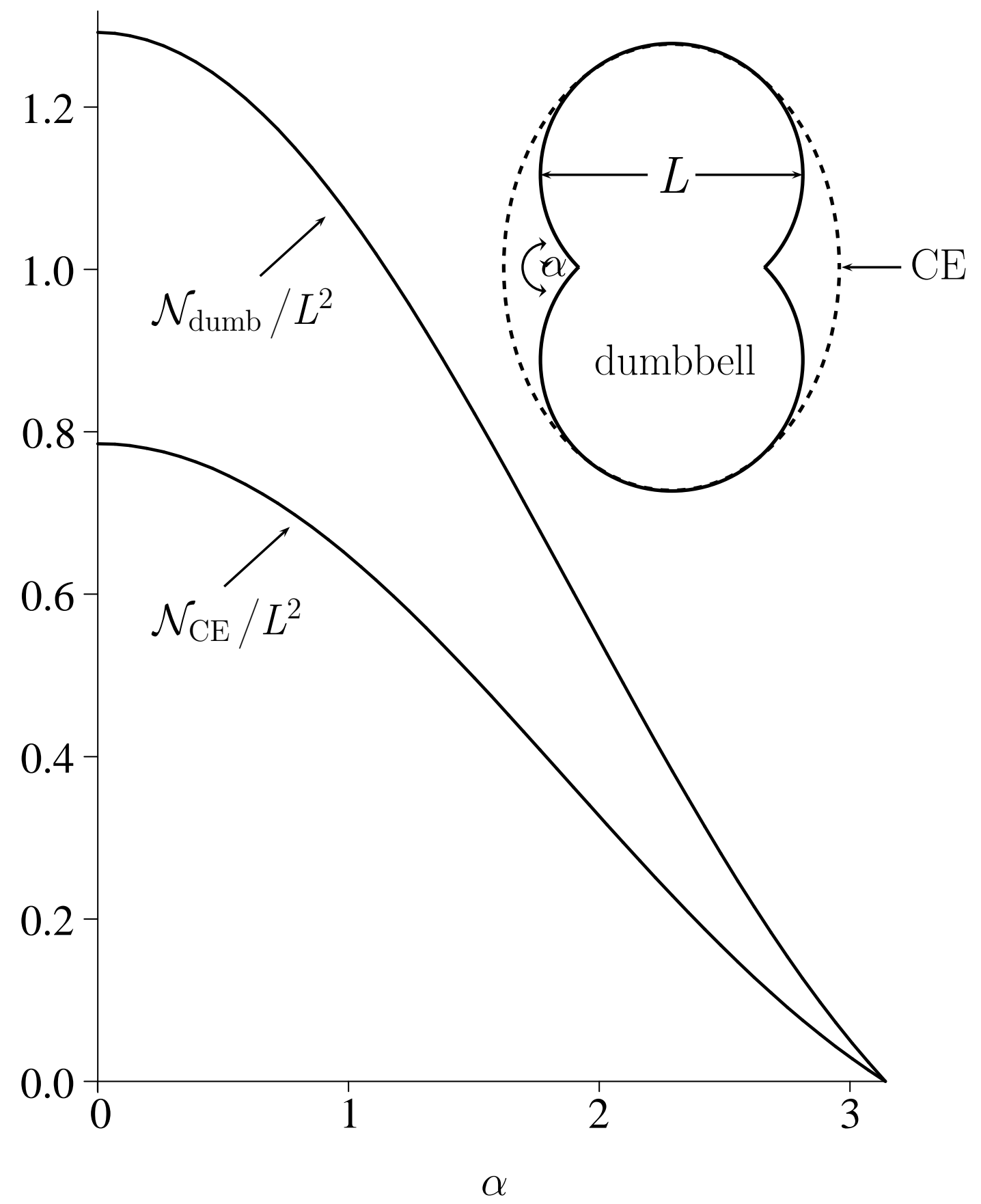

FIG. 2 